\title{
A METHOD FOR SOLVING THE MINIMUM REFLUX PROBLEM OF NONIDEAL MULTICOMPONENT DISTILLATION
}

\author{
IKUHO YAMADA, TAKAITSU IWATA, YOICHI NISHI \\ AND SETSURO HIRAOKA \\ Department of Industrial Chemistry, Nagoya Institute \\ of Technology, Nagoya 466
}

\begin{abstract}
A concept of hypothetical pinch plate which is distinguished from the real pinch point has been proposed to solve the minimum reflux problem of nonideal multicomponent distillation for the operation type. It is assumed that the hypothetical pinch plate takes at a place between the real pinch point and the feed plate in the section where the nondistributed component exists. Any nondistributed component does not exist in the real pinch point, but an allowable small amount of nondistributed component exists in the hypothetical pinch plate. The composition of distillate and bottoms has been calculated by using the component relative volatility evaluated from the liquid composition of the hypothetical pinch plate or the feed plate. Also the normalized $\theta$ 's method has been applied to converge the liquid composition of hypothetical pinch plate and the over-all component material balance.
\end{abstract}

\section{Introduction}

The minimum reflux ratio is an important factor which is a measure of the relative difficulty of a component separation in a distillation process as well as the minimum number of theoretical plates. For the separation of an ideal multicomponent mixture (constant relative volatility throughout the column and constant molar overflow in each section), it is not difficult to determine these factors, but if the system is nonideal (the relative volatilities vary throughout the column), the multicomponent problem is very complicated. For a conventional distillation column which is to operate at a constant pressure and at equimolar vapor and liquid rates in each section of the column, there exist four additional degrees of freedom. To solve the minimum reflux problem, two degrees of freedom are taken to be the specification of infinitely many theoretical plates in each section of the column. The two remaining degrees of freedom can be assigned by either "design type" or "operation type". The design type is to calculate the minimum reflux ratio by assigning molar rates of the light and the heavy key components in distillate and bottoms, whereas the operation type is to calculate the liquid composition of distillate and bottoms by assigning the minimum reflux ratio and the molar rate of distillate or bottoms. The many

Received March 7, 1977. Correspondence concerning this article should be addressed to I. Yamada. T. Iwata is with Mitsui Shipbuilding \& Eng. Co., Ltd., Tokyo 104. attempts ${ }^{1,4,6)}$ to carry out the design type by means of an analytical method, the work of this type can be represented by Underwood's method ${ }^{6}$. On the other hand, a numerical method for solving the operation type was suggested by Holland ${ }^{2)}$ and an analytical method by Yamada et al. ${ }^{8}$. Except for those described by Holland ${ }^{2}$, all methods mentioned above are limited to the ideal system. Sugie et al. ${ }^{5}$, however, suggested that the method used in the ideal system can be applied to the nonideal system only when all components contained in the feed are distributed into both distillate and bottoms.

In this paper, a new calculation method is proposed for solving minimum reflux problems of the operational type for nonideal systems by combining the numerical method by use of the concept of hypothetical pinch plate with the analytical method. This method is illustrated by numerical examples.

\section{Assignment of Key Component and Pinch Point Located in Each Section}

It is first assumed that the composition of distillate and bottoms and the key components ( $h$ and $l$ ) are evaluated from the liquid composition at the pinch point $x_{\infty}(i)$ or $x_{\infty} *(i)$ and from the component reciprocal relative volatilities $p_{\infty}(i)$ or $p_{\infty} *(i)$ (these are also can be evaluated from $x_{\infty}(i)$ and $\left.x_{\infty} *(i)\right)$ even though the values of $p(i)$ and $p_{*}(i)$ vary throughout the column). For the given $x_{\infty}(i)$ and $x_{\infty} *(i)$ the relationships between the equations to determine the 
eigenvalues, $\lambda_{k}$ and $\mu_{k}$, and key components, $h$ and $l$, are as follows ${ }^{7}$ :

\section{1 Enriching section}

(a) $k<h$

$$
\sum_{i} \frac{x_{\infty}(i)}{\lambda_{k}-p_{\infty}(i)}=0
$$

where the components are numbered in order of decreasing reciprocal relative volatility $p(i)$. Therefore, $k$ denotes any component more volatile than the heavy key component.

(b) $k>h$

$$
\lambda_{k}=p_{\infty}(k)
$$

where $k$ denotes the nondistributed component in distillate.

(c) $k=h$

$$
\lambda_{h}=\left\{\frac{1}{R_{m}}+\sum_{i} \frac{\xi_{\infty}(i)}{\zeta_{\infty}(i)}\right\}\left\{\sum_{i} \alpha_{\infty}(i) \frac{\xi_{\infty}(i)}{\zeta_{\infty}(i)}\right\}^{-1}
$$

where

$$
\begin{aligned}
& \xi_{\infty}(i) \equiv \prod_{k}(h)\left[\lambda_{k}-p_{\infty}(i)\right] \\
& \zeta_{\infty}(i) \equiv \prod_{k}(i)\left[p_{\infty}(k)-p_{\infty}(i)\right]
\end{aligned}
$$

Furthermore, $\lambda_{h}$ should be held in the following in order.

$$
p_{\infty}(h)<\lambda_{h}<p_{\infty}(h-1)
$$

\section{2 Stripping section}

(a*) $k<l$

$$
\sum_{i} \frac{x_{\infty} *(i)}{p_{\infty}^{*} *(i)-\mu_{k}}=0
$$

where $k$ denotes any component less volatile than the light key component.

(b*) $k>l$

$$
\mu_{r_{t}}=p_{\infty}^{*} *(k)
$$

where $k$ denotes the nondistributed component in bottoms.

$$
\begin{aligned}
& \left(\mathrm{c}^{*}\right) \quad k=l \\
& \mu_{l}=\left\{-\frac{1}{R_{m}^{*}}+\sum_{i} \frac{\xi_{\infty}^{*}(i)}{\zeta_{\infty}^{*} *(i)}\right\}\left\{\sum_{i} \alpha_{\infty}^{*} *(i) \frac{\xi_{\infty}^{*}(i)}{\zeta_{\infty}^{*} *(i)}\right\}^{-1}
\end{aligned}
$$

where

$$
\begin{aligned}
& \xi_{\infty}^{*} *(i) \equiv \prod_{k}(l)\left[p_{\infty}^{*} *(i)-\mu_{k}\right] \\
& \zeta_{\infty}^{*}(i) \equiv \prod_{k}(i)\left[p_{\infty}^{*} *(i)-p_{\infty}^{*} *(k)\right]
\end{aligned}
$$

$\mu_{l}$ should be held in the following limits:

$$
p_{\infty}^{*} *(l)>\mu_{l}>p_{\infty}^{* *}(l+1)
$$

For an $m$-component system, the relationships between existence of nondistributed component and pinch point located in each section are considered in the following cases ${ }^{7}$.

[case 1] $h=1, l=m$ : No nondistributed components exists in either distillate or bottoms. The pinch point in each section is around the feed plate. The

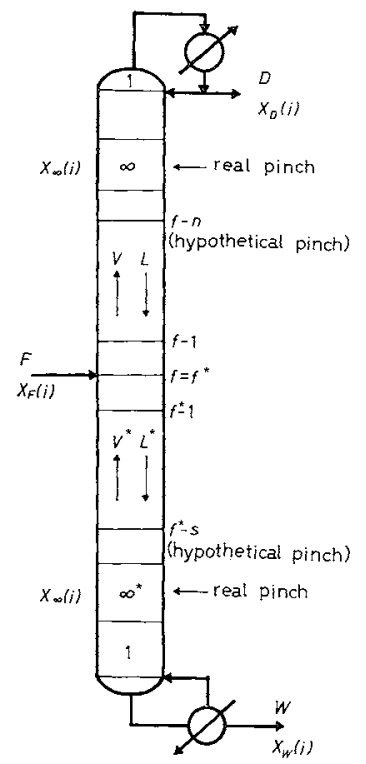

Fig. 1 Schematic diagram of pinch point located in each section for $h \neq 1, l \neq m$

liquid composition on the feed plate equals to that of the liquid portion of feed.

[case 2] $h \neq 1, l=m$ : Nondistributed component exists in distillate but no nondistributed component exists in bottoms. The pinch point in the stripping section is adjacent to the feed plate, but the liquid composition on the feed plate differs from that of the liquid portion of feed. The pinch point in the enriching section is split from the feed plate and moves toward the middle region of the enriching section.

[case 3] $h=1, l \neq m$ : The relationship between existence of nondistributed component and pinch point in each section is the reverse of [case 2].

[case 4] $h \neq 1, l \neq m$ : Nondistributed component exists in both distillate and bottoms, and the pinch points in each section are split from the feed plate. Of course the liquid composition on the feed plate differs from that of the liquid portion of feed.

When the minimum reflux problem of the operation type is solved, neither the liquid composition at the pinch point nor the key components are known in advance. However, by the considereation in [case 1] the existence of nondistributed component is presumed; also, the key component may approximately be assigned by finding $h$ and $l$ which satisfy Eqs. (6) and (12) by the trial and error procedure, using the eigenvalues evaluated from the liquid composition of the liquid portion of feed.

\section{Hypothetical Pinch Plate}

As mentioned in Fig. 1, it is assumed that a hypothetical pinch plate in each section takes place at $(f-n)$-th and $\left(f^{*}-s\right)$-th plate when nondistributed component exists in distillate and bottoms, respec- 
tively. In other words, it takes place at $n$-th or $s$-th plate from the feed plate, where $n$ or $s$ is a finite number depending on the accuracy of solution, respectively. The characteristics of the hypothetical pinch plate are summarized by the following items: i) an infinite number of theoretical plates is considered between the hypothetical pinch plate and the real pinch point; ii) no nondistributed component exists in the real pinch point, but an allowable small amount of nondistributed component exists in the hypothetical pinch plate; iii) the liquid composition in the real pinch is very close to that of the hypothetical pinch plate, therefore $\lim _{n \rightarrow \infty} x_{f-n}(i)=x_{\infty}(i)$ and $\lim _{s \rightarrow \infty} x_{f_{-s}}(i)=$ $x_{\infty} *(i)$.

For convenience, in this paper the hypothetical pinch plate in enriching and stripping section are denoted $j$ and $j^{*}$ instead of $(f-n)$ and $\left(f^{*}-s\right)$, respectively.

\section{Calculation Procedure}

The calculation procedure in the present method is summarized as follows:

1) For an assumed value of liquid composition on the feed plate $x_{f}(i)$, to calculate the terminal composition, $x_{D}(i)$ and $x_{W}(i)$, by using the characteristics of the hypothetical pinch plate.

2) To obtain a corrected $x_{f}(i)$ which satisfies the overall component material balance.

Although only [case 1] was considered, Sugie et al. ${ }^{5 !}$ suggested that $x_{D}(i)$ and $x_{W}(i)$ can be obtained by the eigenvalues evaluated from the real pinch point (in this case $\left.x_{\infty}(i)=x_{\infty} *(i)=x_{F L}(i)\right)$, even though the component relative volatilities vary throughout the column. Thus, one may approximately calculate $x_{D}(i)$ and $x_{W}(i)$ by the eigenvalues evaluated from the hypothetical pinch plate even when nondistributed component exists, since the liquid composition at the hypothetical pinch plate is close to that of the real pinch point as suggested by the characteristics iii). (in this case $x_{\infty}(i)$ and $x_{\infty} *(i)$ differ from $x_{f}(i)$ and $x_{F L}(i)$, respectively.)

For a given value of $x_{f}(i)$ the calculation procedure required to converge the component material balance in each section is as follows:

\section{1 Enriching section}

When the liquid composition on the hypothetical pinch plate, $x_{j}(i)$, is assumed, $\lambda_{k}$ and $h$ are obtained by Eq. (1) to (6), by using the subscript $j$ instead of the subscript $\infty$. Then $x_{D}(i)$ can be calculated by

$$
x_{D}(i)=R_{m} \alpha_{j}(i) \frac{\xi_{j}(i)}{\zeta_{j}(i)}
$$

Using $x_{D}(i)$ and $x_{j}(i)$ obtained by the previous step, the value of $x_{f-1}(i)$ can be calculated by the plate-toplate method, where $x_{D}(i)$ and $x_{f-1}(i)$ calculated must be satisfy the following component material balance equation:

$$
V y_{f}(i)=D x_{D}(i)+L x_{f-1}(i)
$$

where $y_{f}(i)$ can be evaluated from $x_{f}(i)$. Then, $x_{j}(i)$ is corrected by the normalized $\theta$ 's method ${ }^{3)}$. The equation is as follows:

$$
x_{j}(i)_{c o}=\frac{\theta_{j} x_{j}(i)_{a s} V y_{f}(i)}{D x_{D}(i)_{c a l}+L x_{f-1}(i)_{c a l}}
$$

where $\theta_{j}$ is calculated by

$$
\theta_{j}=\left[\sum_{i} \frac{x_{j}(i)_{a s} V y_{f}(i)}{D x_{D}(i)_{c a l}+L x_{f-1}(i)_{c a l}}\right]^{-1}
$$

This calculation process is repeated until a given convergence criterion is satisfied. Also, $\mid x_{\infty}(i)-$ $x_{j}(i) \mid$ for the distributed component must be less than the accuracy desired. Otherwise one must repeat the calculation again with a new $n$ which is larger than the previous one.

Furthermore, it will be useful to take the initial value of $x_{j}(i)$ to be $x_{f}(i)$. If a negative $x_{c}(i)(j+1<$ $c<(f-1))$ is obtained in the plate-to-plate calculation step, $x_{c-1}(i)$ obtained must be used instead of $x_{f-1}(i)$ in Eqs. (15) and (16).

If it is assigned $h=1$, that is, all components contained in the feed are distributed in distillate, $x_{D}(i)$ can be calculated without any consideration of the hypothetical pinch plate. The equation is as follows:

$$
x_{D}(i)=R_{m} \alpha_{f}(i) \frac{\xi_{f}(i)}{\zeta_{f}(i)}
$$

\section{2 Stripping section}

If the liquid composition on the hypothetical pinch plate $x_{j} *(i)$ is given, $\mu_{k}$ and $l$ are obtained by Eqs. (7) to (12), by using the subscript $j^{*}$ instead of the subscript $\infty^{*}$. Then, $x_{W}(i)$ can be calculated by

$$
x_{W}(i)=R_{m}^{*} \alpha_{j^{*}}^{*}(i) \frac{\xi_{j^{*}}^{*}(i)}{\zeta_{j^{*}}^{*}(i)}
$$

For $x_{W}(i)$ and $x_{j} *(i)$ obtained by the previous step, $y_{f} *-1(i)$ can be calculated by the plate-to-plate method. Also, $x_{W}(i)$ and $y_{f *_{-1}}(i)$ must satisfy the following material balance equation:

$$
L^{*} x_{f}(i)=W x_{W}(i)+L^{*} y_{f} *_{-1}(i)
$$

Then $x_{j} *(i)$ is corrected in the same manner as in the enriching section.

$$
x_{j} *(i)_{c o}=\frac{\theta_{j} * x_{j} *(i)_{a s} L^{*} x_{f}(i)}{W x_{W}(i)_{c a l}+V^{*} y_{f}{ }_{-1}(i)_{c a l}}
$$

where $\theta_{j} *$ is calculated by

$$
\theta_{j}=\left[\sum_{i} \frac{x_{j} *(i)_{a s} L^{*} x_{f}(i)}{W x_{W}(i)_{c a l}+V^{*} y_{f *-1}(i)_{c a l}}\right]^{-1}
$$

This calculation process is repeated in the same manner as in the enriching section. Of course, the initial value of $x_{j} *(i)$ and a negative $x_{c} *(i)\left(j^{*}+1<\right.$ $\left.c^{*}<\left(f^{*}-1\right)\right)$ are treated in the same manner as in the enriching section. 
If, $l=m$, that is, no nondistributed component exists in the bottoms, $x_{W}(i)$ is calculated by the following equation:

$$
x_{W}(i)=R_{m}^{*} \alpha_{f}(i) \frac{\xi_{f}^{*}(i)}{\zeta_{f}^{*}(i)}
$$

\section{3 Overall component material balance}

The calculation method mentioned in the previous section is based on $x_{f}(i)$ assumed. Therefore, $x_{f}(i)$ is corrected to satisfy the overall component material balance. The equation is as follows:

$$
x_{f}(i)_{c o}=-\frac{\theta_{f} x_{f}(i)_{a s} F x_{F}(i)}{D x_{D}(i)_{c a l}+W x_{W}(i)_{c a l}}
$$

where $\theta_{f}$ is calculated by

$$
\theta_{f}=\left[\sum_{i} \frac{x_{f}(i)_{a s} F x_{F}(i)}{D x_{D}(i)_{c a l}+W x_{W}(i)_{c a l}}\right]^{-1}
$$

This alternative major calculation loop is repeated until a given convergence criterion has been satisfied. Finally, it is useful to take the initial value of $x_{f}(i)_{\alpha s}$ to be $x_{F L}(i)$.

\section{Numerical Example}

The proposed method is examined with two numerical examples of the ternary system water (1) - methanol (2)-acetone (3) and of the quaternary system 2propanol (1) - ethanol (2) - methanol (3) - ethyl acetate (4). For the first example, the operating condition and the physical property related the vapor-liquid equilibrium are given in Table 1, where the equation for predicting the component activity coefficient in the liquid phase follows the zeroth approximation and the configuration of molecular triplet ${ }^{8}$. The calculated results are shown in Table 2. In this case, the nondistributed component exists in bottoms only, and one can obtain the accurate solution by assigning $s=6$. Table 3 shows the operating condition and the physical property of the second example. Table 4 gives the calculated results for various $n$ and $s$, and shows the existence of the nondistributed component in both distillate and bottoms. One may obtain the solution by $\delta_{\text {max }}\left(\equiv\left|x_{f-n}(i)-x_{\infty}(i)\right|_{\max }\right.$ or $\mid x_{f} *_{-s}(i)-$ $\left.\left.x_{\infty} *(i)\right|_{\max }\right)<0.005$. Furthermore, the initial values of $x_{j}(i), x_{j} *(i)$ and $x_{f}(i)$ in Eqs. (15), (19) and (22) for both examples were taken to be $x_{F L}(i)$. Stable solutions were obtained as observed in Tables 2 and 4 even though $x_{f-n}(i)\left(=x_{j}(i)\right), x_{f} *_{-s}(i)\left(=x_{j} *(i)\right)$ and $x_{f}(i)$ differ greatly from $x_{F L}(i)$. This stable solution probably is one of the characteristics of the present method. The concentration profiles calculated for both examples are shown in Figs. 2 and 3. These figures demonstrate the consistency of the first assumption in which $x_{D}(i), x_{W}(i)$ and key components, $h$ and $l$, can be evaluated by the liquid composition and the component reciprocal relative volatilities at the pinch point or the hypothetical pinch plate.

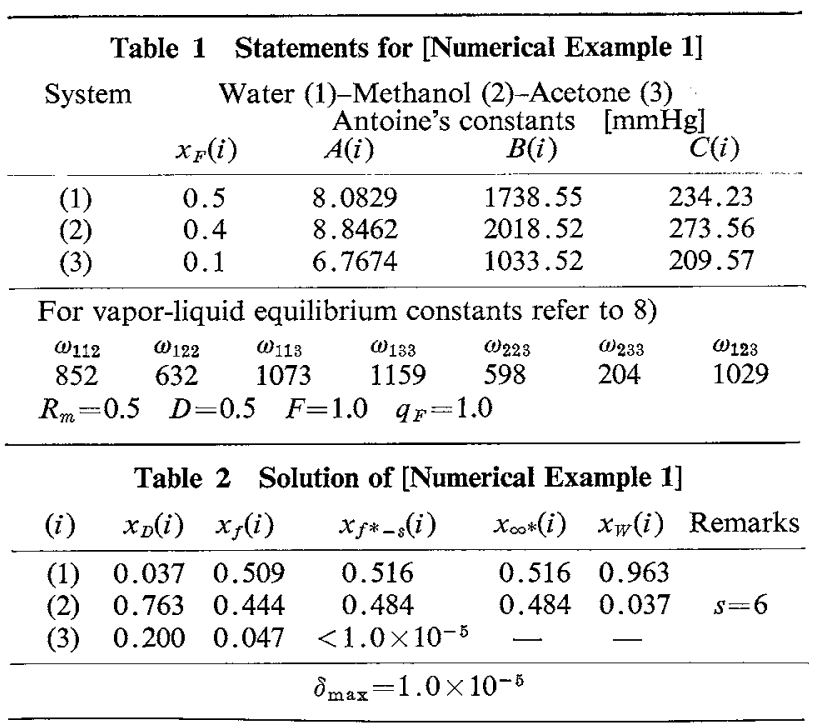

Table 3 Statements for [Numerical Example 2]

System 2-Propanol (1)-Ethanol (2)-Methanol (3)Ethyl acetate (4)

\begin{tabular}{ccccc} 
& \multicolumn{3}{c}{ Ethyl acetate (4) } & \multicolumn{2}{c}{ Antoine's constants } & {$[\mathrm{mmHg}]$} \\
& $x_{F}(i)$ & $A(i)$ & $B(i)$ & $C(i)$ \\
\hline$(1)$ & 0.35 & 8.9657 & 2063.43 & 256.57 \\
$(2)$ & 0.10 & 7.8312 & 1440.52 & 212.71 \\
$(3)$ & 0.45 & 8.8462 & 2018.53 & 273.56 \\
$(4)$ & 0.10 & 7.2235 & 1306.26 & 223.67 \\
\hline
\end{tabular}

For vapor-liquid equilibrium constant refer to 8 )

$$
\begin{array}{cccc}
\omega_{114}=487 & \omega_{144}=321 & \omega_{224}=616 \quad \omega_{244}=581 \\
\omega_{334}=825 & \omega_{344}=558 & \\
\omega_{124}=501 & \omega_{134}=548 \quad \omega_{234}=645 & \\
\text { other } \omega_{i i j}, \omega_{i j j}, \omega_{j j k} \text { 's are all zero } & \\
R_{m}=1.5 \quad D=0.55 \quad F=1.0 \quad q_{F}=1.0
\end{array}
$$

Table 4 Solution of [Numerical Example 2]

\begin{tabular}{lcccrccc} 
(i) & $x_{D}(i)$ & $x_{\infty}(i)$ & $x_{f-n}(i)$ & $x_{f^{*-s}}(i)$ & $x_{\infty} *(i)$ & $x_{W}(i)$ & Remarks \\
\hline (1) & - & - & 0.053 & 0.363 & 0.363 & 0.778 & \\
(2) & 0.025 & 0.318 & 0.288 & 0.111 & 0.111 & 0.192 & $n=21$ \\
(3) & 0.793 & 0.617 & 0.596 & 0.525 & 0.526 & 0.030 & $s=11$ \\
(4) & 0.182 & 0.065 & 0.063 & $<0.001$ & - & - & \\
(1) & - & - & 0.005 & 0.363 & 0.363 & 0.778 & \\
(2) & 0.024 & 0.318 & 0.316 & 0.112 & 0.112 & 0.193 & $n=31$ \\
(3) & 0.794 & 0.619 & 0.617 & 0.524 & 0.525 & 0.029 & $s=11$ \\
(4) & 0.182 & 0.063 & 0.062 & $<0.001$ & - & - & \\
\hline
\end{tabular}

\section{Discussion and Conclusion}

A calculation method has been proposed to solve the minimum reflux problem of the operational type for nonideal systems, and the important characteristics of the proposed method can be summarized as follows:

[i] The heavy and light key components can be found by evaluating the eigenvalues from the liquid composition at the hypothetical pinch point.

[ii] For the section where the nondistributed component exists, the hypothetical pinch plate is considered in addition of the real pinch point, where a finite number of theoretical plates is considered between the hypothetical pinch plate and the feed plate, whereas there are infinitely many plates between the two pinches. 

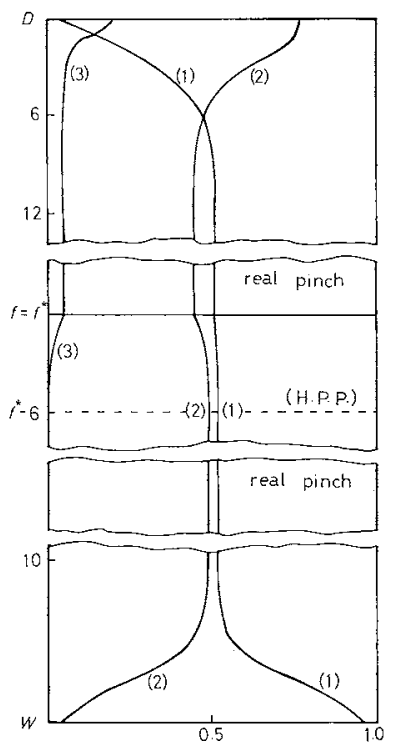

Fig. 2 Concentration profile for numerical example 1

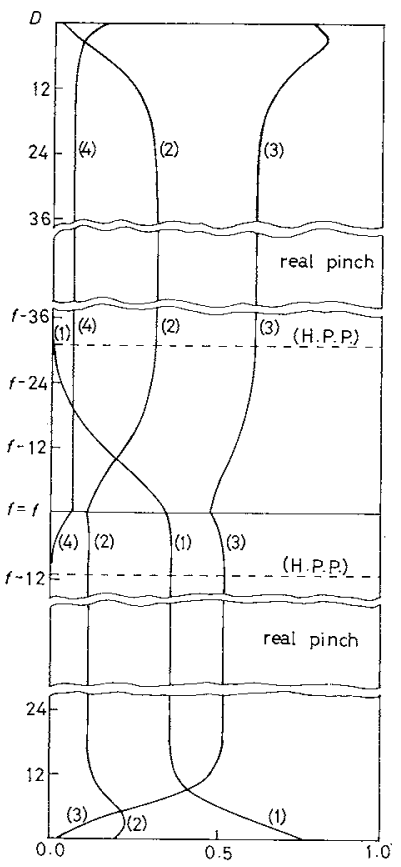

Fig. 3 Concentration profile for numerical example 2 $(n=31, s=11)$

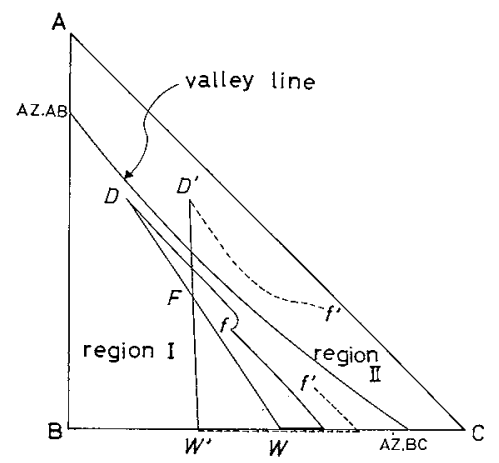

Fig. 4 Comparison of tieline calculated with reflux ratio [iii] The terminal composition such as $x_{D}(i)$ and $x_{W}(i)$ is calculated by use of $p(i)$ evaluated from the liquid composition of the feed plate for the section where no nondistributed component exists and from the liquid composition of the hypothetical pinch plate where the nondistributed component exists, even though $p(i)$ varies throughout the column. Therefore, a consistent solution cannot be obtained unless the tieline calculated does not lie across the valley or the ridge line. This is the most important feature of the proposed method.

Generally, the calculated tieline for the minimum reflux of the operational type is affected by the reflux ratio. As illustrated in Fig. 4, for a ternary system with the two regions, I and II, divided by the valley line characterized the vapor-liquid equilibrium, the calculated tieline by the proposed method may move from $\overline{D F W}$ to $\overline{D^{\prime} F W^{\prime}}$ lying across the valley line as the reflux ratio increases in accordance with the characteristics suggested in [iii]. (In this case $x_{D}(i)$ is calculated by use of $p(i)$ evaluated from $x_{f}(i)$ since no nondistributed component exists in the distillate.) To obtain a consistent solution of the distillation problem the condition of "feed plate matching" must be satisfied, that is, the liquid composition on the feed plate calculated from the composition of distillate is equal to that from the bottoms. It is obvious that the locus of the liquid composition on the plate in the enriching section calculated from the distillate composition located in the region II never lies across the valley line, as shown by the curve $\overparen{D^{\prime} f^{\prime}}$ in Fig. 4. Therefore, the calculated tieline $\overline{D^{\prime} F W^{\prime}}$ never holds for the condition of "feed plate matching". On the other hand the tieline $\overline{D F W}$ holds for this condition since the locus of the liquid composition on the plate in each section is at and adjacent to the feed plate. The same consideration mentioned above may be applicable to a ternary system with the ridge line. Thus, the proposed method fails to obtain a consistent solution when the calculated tieline lies across the valley or the ridge line. In this case one may obtain an approximately solution by assigning a sufficiently large number of theoretical plates in the section where the tieline lies across the valley or the ridge line.

On the other hand, the characteristics suggested in [ii] implies that one may obtain a more accurate solution by increasing $n$ and $s$. However, a satisfactory result will be obtained by giving $n$ or $s$ a value of fewer than 31 plates.

Furthermore, it should be pointed out the normalized $\theta$ 's method shown in Eqs. (16), (20) and (22) is applied to obtain the converged solution as an additional characteristic. 


\section{Acknowledgment}

The authors wish to acknowledge the continuing encouragement of Professor C. D. Holland, Texas A. \& M. University, and Mrs. Holland.

\section{Nomenclature}

\begin{tabular}{|c|c|c|}
\hline \multicolumn{2}{|c|}{$A(i), B(i), C(i)=$ Cox-Antoine's constant } & {$[-]$} \\
\hline$D$ & $=$ molar rate of distillate & {$[\mathrm{mol} / \mathrm{hr}]$} \\
\hline$F$ & $=$ molar rate of feed & {$[\mathrm{mol} / \mathrm{hr}]$} \\
\hline$L$ & $\begin{array}{l}=\text { molar rate of liquid through the } \\
\text { column }\end{array}$ & {$[\mathrm{mol} / \mathrm{hr}]$} \\
\hline$p(i)$ & $\begin{array}{l}=\text { reciprocal relative volatility of } \\
\text { component } i\end{array}$ & {$[-]$} \\
\hline $\boldsymbol{R}_{m}$ & $=$ minimum reflux ratio & {$[-]$} \\
\hline$V$ & $\begin{array}{l}=\text { molar rate of vapor through the } \\
\text { column }\end{array}$ & {$[\mathrm{mol} / \mathrm{hr}]$} \\
\hline$W$ & $=$ molar rate of bottoms & {$[\mathrm{mol} / \mathrm{hr}]$} \\
\hline$x(i)$ & $=$ molar fraction of liquid of component $i$ & {$[-]$} \\
\hline$y(i)$ & $=$ molar fraction of vapor of component $i$ & {$[-]$} \\
\hline$\alpha(i)$ & $=$ component relative volatility & {$[-]$} \\
\hline$\zeta(i)$ & $=$ defined by Eq. (5) & \\
\hline$\zeta^{*}(i)$ & $=$ defined by Eq. (11) & \\
\hline$\theta$ & $=$ correction factor & {$[-]$} \\
\hline$\lambda$ & $=$ eigenvalue of Eq. (1) & \\
\hline$\mu$ & $=$ eigenvalue of Eq. (7) & \\
\hline$\xi(i)$ & $=$ defined by Eq. (4) & \\
\hline$\xi^{*}(i)$ & $=$ defined by Eq. (10) & \\
\hline$\omega$ & $\begin{array}{l}=\text { constant in equation of liquid activity } \\
\text { coefficient }\end{array}$ & $\left./ /{ }^{\circ} \mathrm{C} \cdot \mathrm{mol}\right]$ \\
\hline
\end{tabular}

\section{〈Subscripts〉}

as = assume

cal $\quad=$ calculated

co $\quad=$ corrected

$D \quad=$ distillate

$F \quad=$ feed

$F L=$ liquid portion of feed

$f \quad=$ feed plate

$j \quad=$ hypothetical pinch plate in enriching section

$j^{*} \quad=$ hypothetical pinch plate in stripping section

$k \quad=k$-th eigenvalue

$W \quad=$ bottoms

$\infty \quad=$ real pinch in enriching section

$\infty^{*} \quad=$ real pinch in stripping section

* $\quad=$ stripping section

\section{Literature Cited}

1) Acrivos, A. and N. R. Amundson: Chem. Eng. Sci., 4, 68 (1955).

2) Holland, C. D.: "Multicomponent Distillation", p. 247 (Prentice-Hall Inc. 1963).

3) Iwata, T., Y. Sato, M. Nakamura, et al.: Kagaku Kogaku Ronbunshu, 3, 311 (1977).

4) Murdoch, P. G. and C. D. Holland: Chem. Eng. Progr., 48, 287 (1952).

5) Sugie, H. and I. Yamada: Kagaku Kōgaku, 31, 1001 (1967).

6) Underwood, A. J. V.: Ind. Eng. Chem., 41, 2844 (1949).

7) Yamada, I., H. Sugie and T. Iwata: Kagaku Koggaku, 31, 565 (1967).

8) Yamada, I., T. Yoshida and S. Haruyama: ibid., 25, 158 (1961). 